\title{
Formation Flying System Design for a Planet-Finding Telescope-Occulter System
}

\author{
Jesse Leitner \\ Code 591, NASA GSFC, Greenbelt, MD, USA;
}

\begin{abstract}
The concept of flying an occulting shade in formation with an orbiting space telescope to enable astronomical imaging of faint targets while blocking out background noise primarily from starlight near distant Earth-like planets has been studied in various forms over the past decade. Recent analysis has shown that this approach may offer comparable performance to that provided by a space-based coronagraph with reduced engineering and technological challenges as well as overall mission and development costs. This paper will present a design of the formation flying architecture (FFA) for such a collection system that has potential to meet the scientific requirements of the National Aeronautics and Space Administration's (NASA's) Terrestrial Planet Finder mission. The elements of the FFA include the relative navigation, intersatellite communication, formation control, and the spacecraft guidance, navigation, and control (GN\&C) systems. The relative navigation system consists of the sensors and algorithms to provide necessary range, bearing or line-of-sight, and relative attitude between the telescope and occulter. Various sensor and filtering (estimation) approaches will be introduced. A formation control and GN\&C approach will be defined that provides the proper alignment and range between the spacecraft, occulter, and target to meet scientific objectives. The state of technology will be defined and related to several formation flying and rendezvous spacecraft demonstration missions that have flown.
\end{abstract}

Keywords: formation flying, space telescope, occulter, planet-finding, spacecraft GN\&C

\section{INTRODUCTION}

The concept of flying an occulting shade in formation with an orbiting space telescope to enable astronomical imaging of faint targets while blocking out background noise primarily from starlight near distant Earth-like planets has been studied in various forms over the past decade. Recent analysis has shown that this approach may offer comparable performance to that provided by a space-based coronagraph with reduced engineering and technological challenges as well as overall mission and development costs. This paper will present a design of the formation flying architecture (FFA) for such a collection system that has potential to meet the scientific requirements of the National Aeronautics and Space Administration's (NASA's) Terrestrial Planet Finder mission. In this paper, the concept will be termed the Terrestrial Planet Finder - Occulter (TPF-O). The paper will begin by discussing the relative navigation system used to measure the relative states between the two vehicles, including sensors and algorithms. Next the concept for intersatellite communication will be presented. Following that, the design of the two-spacecraft formation will be discussed, such that minimum science requirements are met for a "fuel-enabled" highly maneuvering mission. Finally, a concept for controlling the formation will be presented, with the most stringent objective being the control of the alignment of the telescope and occulter with the astronomical target. The key formation flying problem during science collection can be defined in relation to the cartoon illustrated in Figure 1. The telescope shall maintain a range $(\rho)$ of $72000 \mathrm{~km}$ from an occulting star shade (occulter), while the occulter is held in the line-of-sight from the telescope to the target. The angle $\theta$ represents the telescope pointing error, which is stringent, but it represents a well-known problem in telescope attitude control and will not be discussed further here. The angle $\gamma$ represents the offset of the occulter from the telescope focal line, and the primary scientific metric is the angle $\alpha$, which represents the alignment error, which ideally should be zero. The requirements for each will be discussed in the following sections, along with technological solutions to enable the mission.

Further author information: (Send correspondence to Jesse Leitner)

E-mail: jesse.leitner "AT"nasa.gov, Telephone: 13012862630

This is a declared work of the U.S. Government and not subject to copyright in the United States of America.

UV/Optical/IR Space Telescopes: Innovative Technologies and Concepts III edited by Howard A MacEwen James B. Breckinridge

Proc. of SPIE Vol. 6687, 66871D, (2007) · 0277-786X/07/\$18 - doi: 10.1117/12.731626

Proc. of SPIE Vol. 6687 66871D-1 


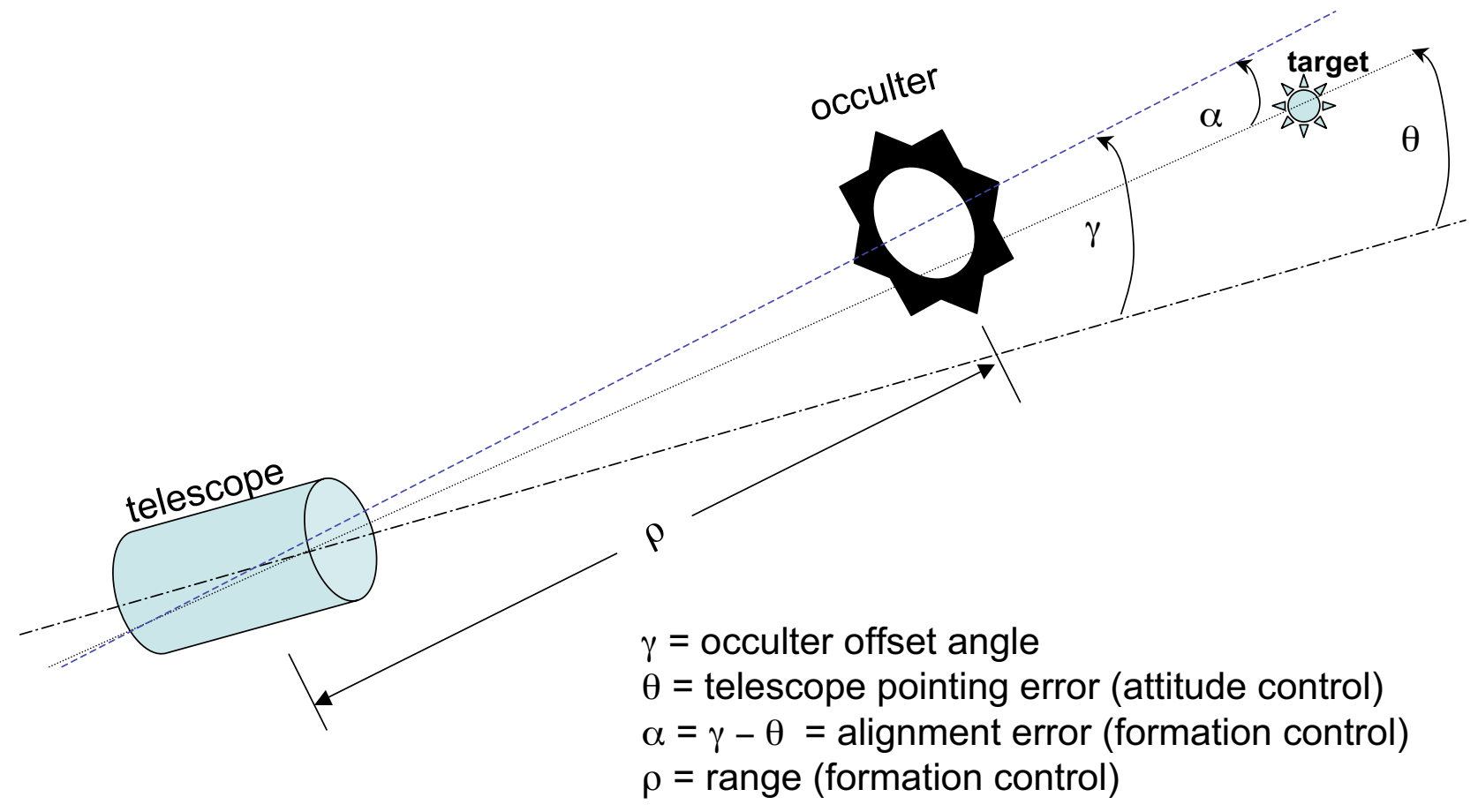

Figure 1. Formation Flying Alignment Diagram.

\section{FORMATION FLYING SYSTEM}

The formation flying system (FFS) is the glue that combines the functions over multiple vehicles into an integrated system, or virtual platform. The FFS comprises sub-systems for relative navigation, intersatellite communication, and formation control. The current set of formation flying mission requirements are expressed in Table 1 , the particularly challenging one being the alignment of the telescope and the occulter relative to the target. As with any feedback control system with a wide capture range or dynamic range and tight control requirements, many modes or stages may be required to transition the multi-vehicle configuration from an uncoupled or loose configuration down to a precisely controlled virtual platform. These modes can involve multiple sensors and actuators, estimation and control algorithms, and potentially complex mode-switching logic. Table 2 lists a likely set of modes that will exist within the FFS, along with the entrance criteria for each mode.

\subsection{Relative Navigation}

The process of determining relative states between vehicles using data gathered from sensors or the science instrument itself will be termed relative navigation. The relative states include positions, velocities, angles, angular rates, or alternatively, range and bearing and associated rates. The relative navigation subsystem includes the sensors, algorithms, and software required. There are multiple stages in the current relative navigation subsystem design, ranging from coarse vehicle-to-vehicle range measurement in the vicinity of the science orbit (handed off from the absolute nav of the individual vehicles) through fine relative state measurement required to control the range and line-of-sight between the telescope and occulter. Table 3 presents several candidate hardware approaches to cover the range of coarse navigation upon orbit insertion through the finest science mode stage, including key details for each, such as whether the approach provides absolute (inertial) or relative (spacecraft to spacecraft) navigation, the accuracy of the approach, the measurement bandwidth and latency, and the quantity each measures. ${ }^{1}$ While the first five are mature capabilities, ${ }^{2}$ the last two represent a very 
Table 1. Formation flying requirements.

\begin{tabular}{|c|c|c|}
\hline & Requirement & Source \\
\hline \multicolumn{3}{|l|}{ Key Requirements } \\
\hline Mission Life (on station) & 36-60 months & $\begin{array}{c}\text { Design reference mission } \\
(\mathrm{DRM})\end{array}$ \\
\hline $\begin{array}{l}\text { \# observations/duration } \\
\text { of each }\end{array}$ & $70+/ 3$ hrs -5 days & DRM \\
\hline $\begin{array}{l}\text { Integration times for } \\
\text { each image }\end{array}$ & $1 \mathrm{hr}$ & Optical performance \\
\hline $\begin{array}{l}\text { Range measurement } \\
\text { precision }\end{array}$ & $10 \mathrm{~km}$ & $\begin{array}{c}\text { Minimum required for range } \\
\text { control }\end{array}$ \\
\hline $\begin{array}{l}\text { Alignment error } \\
\text { measurement precision }\end{array}$ & 300 microarcsecond & $\begin{array}{c}\text { Minimum required for } \\
\text { alignment error control }\end{array}$ \\
\hline Range control precision & $100 \mathrm{~km}$ & Little sensitivity in range \\
\hline $\begin{array}{l}\text { Alignment error control } \\
\text { precision }\end{array}$ & 1 mas & $\begin{array}{c}\text { Several centimeters lateral } \\
\text { offset at } 72000 \mathrm{~km}\end{array}$ \\
\hline $\begin{array}{l}\text { Max range between } \\
\text { telescope \& occulter }\end{array}$ & $72000 \mathrm{~km}$ & $\begin{array}{c}\text { Limited by } \Delta v \& \text { required } \\
\text { maneuver times }\end{array}$ \\
\hline
\end{tabular}

Table 2. Formation flying modes with entrance and exit criteria.

\begin{tabular}{|l|l|l|}
\hline \multicolumn{1}{|c|}{ Mode } & \multicolumn{1}{|c|}{$\begin{array}{c}\text { Range Control } \\
\text { Entrance Criterion }\end{array}$} & \multicolumn{1}{|c|}{$\begin{array}{c}\text { Alignment Control } \\
\text { Entrance Criterion }\end{array}$} \\
\hline Insertion & $10,000 \mathrm{~km}$ & $4 \pi$ ster \\
\hline Lost in space & $2,000 \mathrm{~km}$ & $4 \pi$ ster \\
\hline Formation Acquisition & $1,000 \mathrm{~km}$ & $1-5 \mathrm{deg}$ \\
\hline $\begin{array}{l}\text { Configuration } \\
\text { Initialization/Reconfig }\end{array}$ & $200 \mathrm{~km}$ & $<1 \mathrm{deg}$ \\
\hline $\begin{array}{l}\text { Coarse Science Mode } \\
\text { Acquisition }\end{array}$ & $100 \mathrm{~km}$ & $\operatorname{arcsec}$ \\
\hline Calibration & $100 \mathrm{~km}$ & $<0.1 \operatorname{arcsec}$ \\
\hline $\begin{array}{l}\text { Fine Science Mode } \\
\text { Acquisition }\end{array}$ & $100 \mathrm{~km}$ & $50 \mathrm{mas}$ \\
\hline $\begin{array}{l}\text { Science Mode } \\
\text { (maintenance) }\end{array}$ & $100 \mathrm{~km}$ & $5 \mathrm{mas}$ \\
\hline
\end{tabular}


Table 3. Formation flying measurement devices.

\begin{tabular}{|c|c|c|c|c|c|}
\hline Device & Absolute/relative & Accuracy/precision & $\begin{array}{c}\text { Measurement } \\
\text { Bandwidth }\end{array}$ & Latency & Dimension/quantity \\
\hline Ground/Doppler/DSN & Absolute & $50 \mathrm{~km}$ & $1 / 2$ weeks & weeks & Inertial position \\
\hline Celestial Navigation & $\begin{array}{c}\text { Absolute, } \\
\text { relative }\end{array}$ & $\begin{array}{c}50 \mathrm{~km} / 100 \mathrm{~m} / 5 \\
\mathrm{deg}\end{array}$ & $>1 \mathrm{~Hz}$ & $30 \mathrm{sec}$ & $\begin{array}{c}\text { Inertial position, } \\
\text { range, alignment }\end{array}$ \\
\hline Camera & Relative & $50 \mathrm{~m} \mathrm{rms}$ & $\mathrm{mHZ}$ & minutes & range \\
\hline $\begin{array}{c}\text { Beacon/quad cells } \\
\text { Relative }\end{array}$ & $\begin{array}{c}50 \mathrm{~cm} \mathrm{rms} \\
\text { arcmin rms }\end{array}$ & $1 \mathrm{~Hz}$ & $0.1 \mathrm{sec}$ & Range \& alignment \\
\hline $\begin{array}{c}\text { RF interspacecraft } \\
\text { range }\end{array}$ & Relative & $5 \mathrm{~m}$ & $1 \mathrm{~Hz}$ & $0.1 \mathrm{sec}$ & $\begin{array}{c}\text { Range, possibly } \\
\text { alignment }\end{array}$ \\
\hline $\begin{array}{c}\text { Beacons with pulse } \\
\text { code modulation and } \\
\text { high-precision star } \\
\text { tracker }\end{array}$ & Relative & $10 \mathrm{arcmin}$ & $1 \mathrm{~Hz}$ & $0.5 \mathrm{sec}$ & alignment \\
\hline $\begin{array}{c}\text { Wavefront } \\
\text { sensing/phase } \\
\text { retrieval }\end{array}$ & Relative & $1 \mathrm{mas}$ & $0.1 \mathrm{~Hz}$ & $1 \mathrm{sec}$ & alignment \\
\hline
\end{tabular}

precise and sensitive combination of an advanced star tracker, pulse-code-modulated beacons, the occulter, and the telescope as illustrated in Figure 2 followed by a wavefront sensing scheme that uses part of the science image to determine the errors on an optical scale. This process, required to provide alignment measurements on the order of milli-arcseconds is one of the tall-poles of this mission. The figure illustrates the fine alignment sensing information picked off from the science light and the illustrations below show the beacon configuration on the occulter for a sixteen petal example, the image for planet situated at $20 \%$ inside the edge of the field of view. While the list is not all-encompassing, the selection of approaches is based on the fact that the requirements for range measurement and control are very loose, while alignment is the critical parameter. Hence, optical crosslinks, while likely beneficial from a power perspective, may be undesirable due to the associated requirements on vehicle pointing stability, as well as potential stray light interference with the telescope, depending on the configuration. Fortunately, most of the measurement requirements are fairly loose, but the stringent alignment requirement might end up driving the other measurements to precise values based on observability.

\subsection{Intersatellite Communication}

The subsystem enabling communication between the telescope vehicle and the occulter is termed the intersatellite communication system (ICS). The ICS includes hardware, such as radios and antennae, the network and protocols, and any software required for implementation. The key challenges in this area for this mission concept are the robustness of the communication such that closed-loop formation control can be maintained over the $72000 \mathrm{~km}$ distance between spacecraft, under potentially tight power constraints (which are not yet defined) and the ability to continuously measure coarse range between the vehicles even under a range of orientations at $72000 \mathrm{~km}$ separation. Currently an S-band system is baselined primarily due to existing technology solutions that have flown in space. However, trades will be considered between low-frequency (e.g., UHF band) for better omni-directional capabilities, and such higher frequency (e.g., S-band) approaches for better directional performance. The selection will depend on the combination of the power budget with the closed-loop formation control performance requirements, specifically the bandwidth needed for acceptable science performance, particularly in the alignment control budget. 


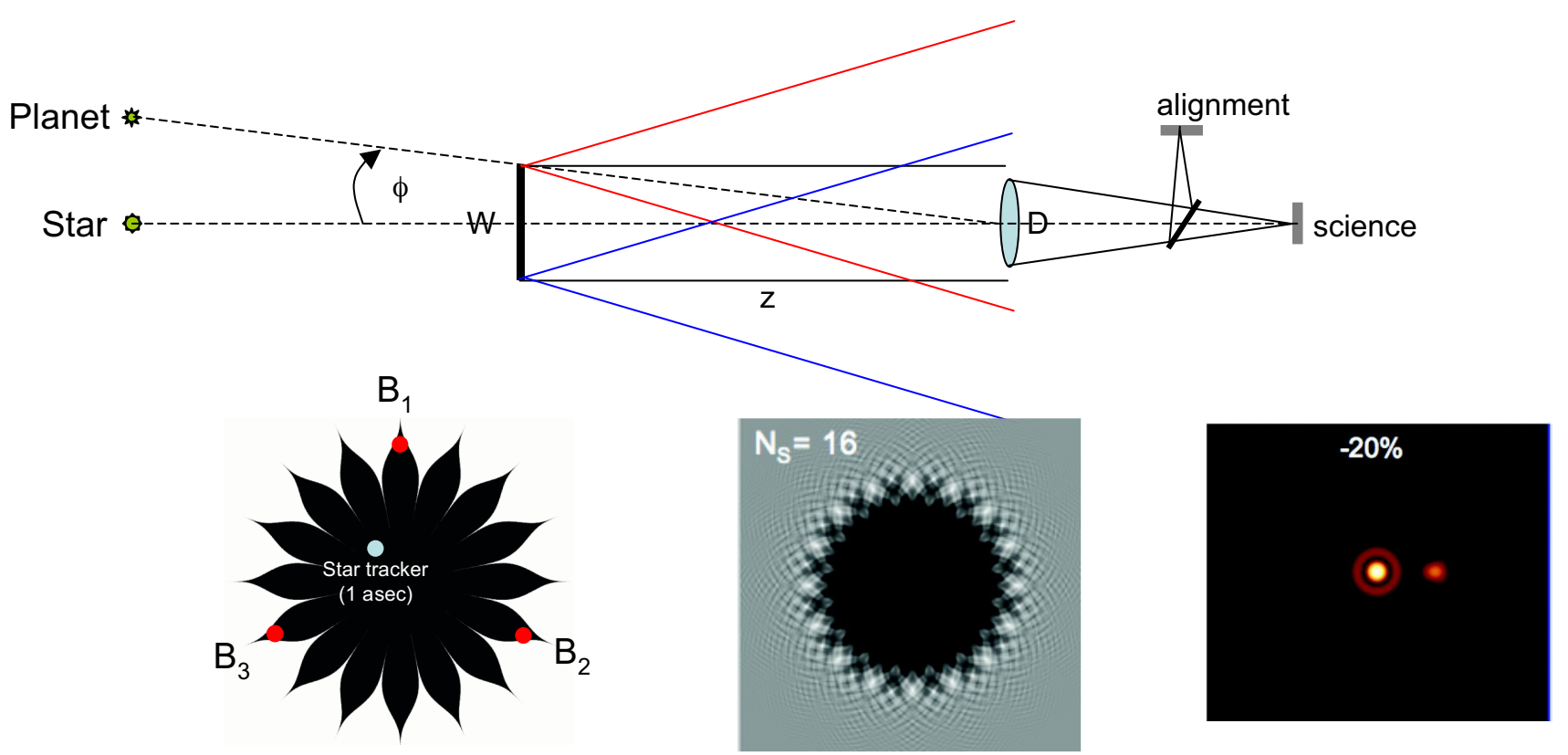

Figure 2. Science mode alignment scheme.

\subsection{Formation Design}

The process of designing the relative motion trajectories to best meet science requirements while optimizing quantities such as fuel efficiency, transfer time, operational complexity, etc., is denoted formation design. This amounts to the formation flying guidance, which considers such quantities as gravity gradient, differential solar pressure and solar wind, minimum and maximum times for staring at a target and reconfiguring a target, and the type of propulsion system. An optimization is performed on the open-loop system to best meet the set of conflicting objectives with proper weighting on the most important constraints. Several orbits are considered, generally Sun-Earth L2 libration point Lissajous and Halo orbits. While the gravity gradient is small in both cases, with a $72000 \mathrm{~km}$ "moment arm" the fuel costs must be considered and nominal orbits become important for a mission of three or more years with frequent reconfigurations. Because the mission concept involves frequent "repointing" of the telescope-occulter pair, this is a "fuel-enabled" mission rather than one where a small percentage of the overall vehicle wet mass is allocated to fuel for stationkeeping and reconfiguration. Fuel consumption is addressed in the next section.

\subsection{Formation Control}

The formation control subsystem brings together all of the components of the formation flying system along with spacecraft GN\&C subsystems with the ultimate goal of maintaining the proper "shape" of the formation to meet the science requirements. Figure 3 shows the integrated formation flying and GN\&C system for the TPF-O mission concept. Aside from the multi-vehicle aspect, some of the unique attributes for this system as compared to single spacecraft and typical constellation missions are the following:

1. An autonomous Attitude and Orbit Control System (AOCS) is in place to handle the six-degree of freedom (6DOF) control of the vehicle, as opposed to simply an attitude control system with orbit corrections from the ground

2. Relative sensors between the vehicles, as for measuring relative position and boresight

3. Processor distributed over both vehicles 


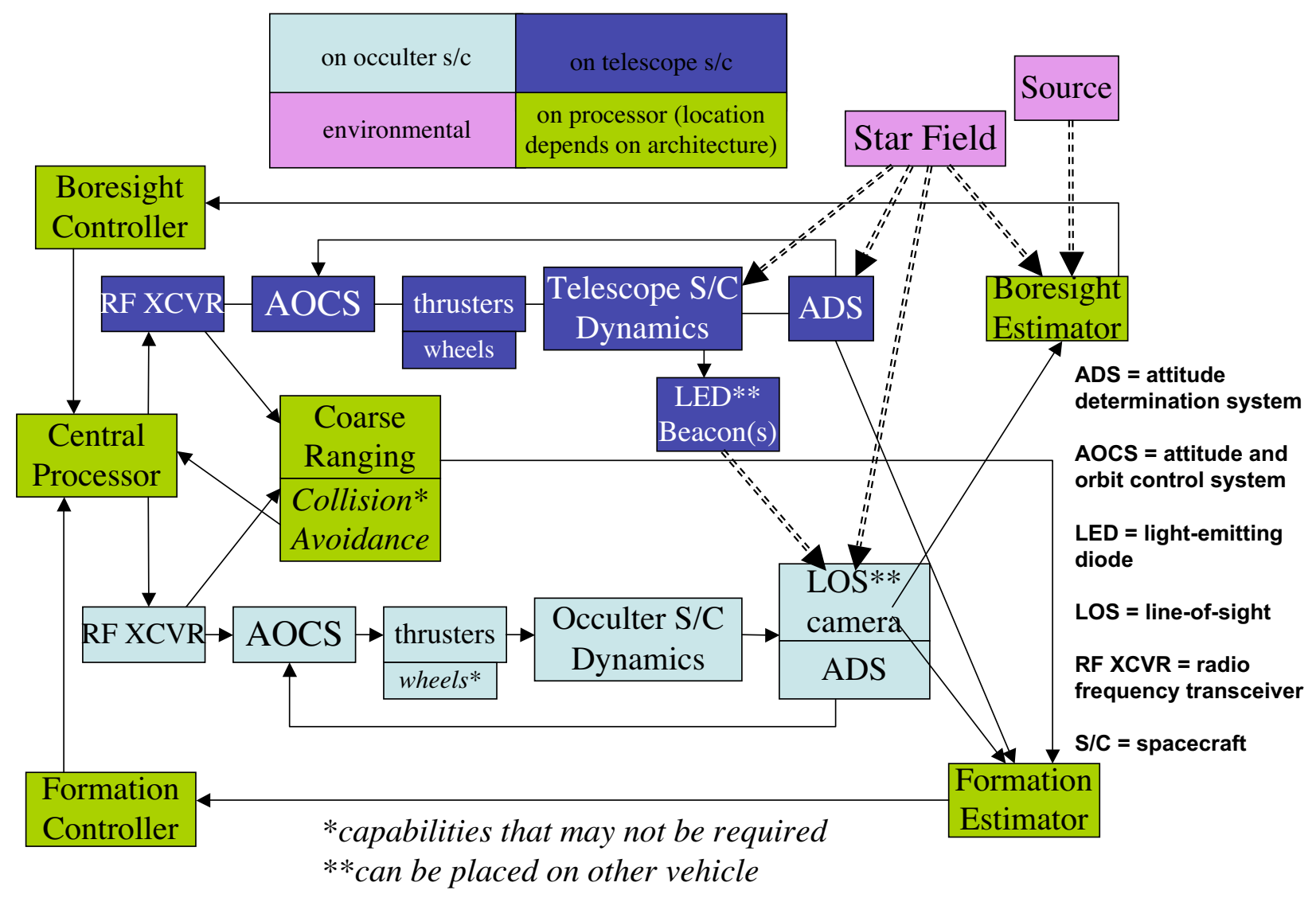

Figure 3. Block diagram for Formation Flying and GNC Subsystem.

4. Intersatellite communication devices in the formation control loop

There are several trades to be performed in the formation control area. At the moment, the priorities are: (1) low-thrust vs impulsive vs hybrid (multi-stage), which then leads into the guidance profiles for reconfiguration (bang-bang, smoothly varying thrust, or bang-coast-bang); (2) type of control algorithm ${ }^{3} ;(3)$ autonomy/level of ground-interaction; and (4) level of control authority and precision during reconfiguration maneuvers. Fuel vs science performance/number of targets accessible will be the primary consideration for most of these trades.

Several studies have been performed to assess fuel consumption, thrusting times, and coasting times for a design reference mission (DRM) with a five year duration (above the minimum three year requirement). Figure 4 shows the cumulative thrusting durations as a function of constant acceleration thrusting levels over the mission duration. Figure 5 shows the overall $\Delta V$ of just over $6.5 \mathrm{~km} / \mathrm{sec}$, which estimates the fuel consumption for the reconfigurations over the five year duration. This is without optimization, but is reasonable to consider for a maneuver-intensive formation flying flagship mission. While the figure is high for a mission for a spacecraft stabilized in an orbit, it is a reasonable figure for a long-duration mission which involves frequent, large-scale spatial maneuvers with time constraints.

\section{FORMATION FLYING TECHNOLOGY STATUS}

In examining the feasibility of the mission concept defined in this paper, it is important to consider the technology capability areas within and their maturity, both in general, and relative to the TPF-O mission concept. Table 4 


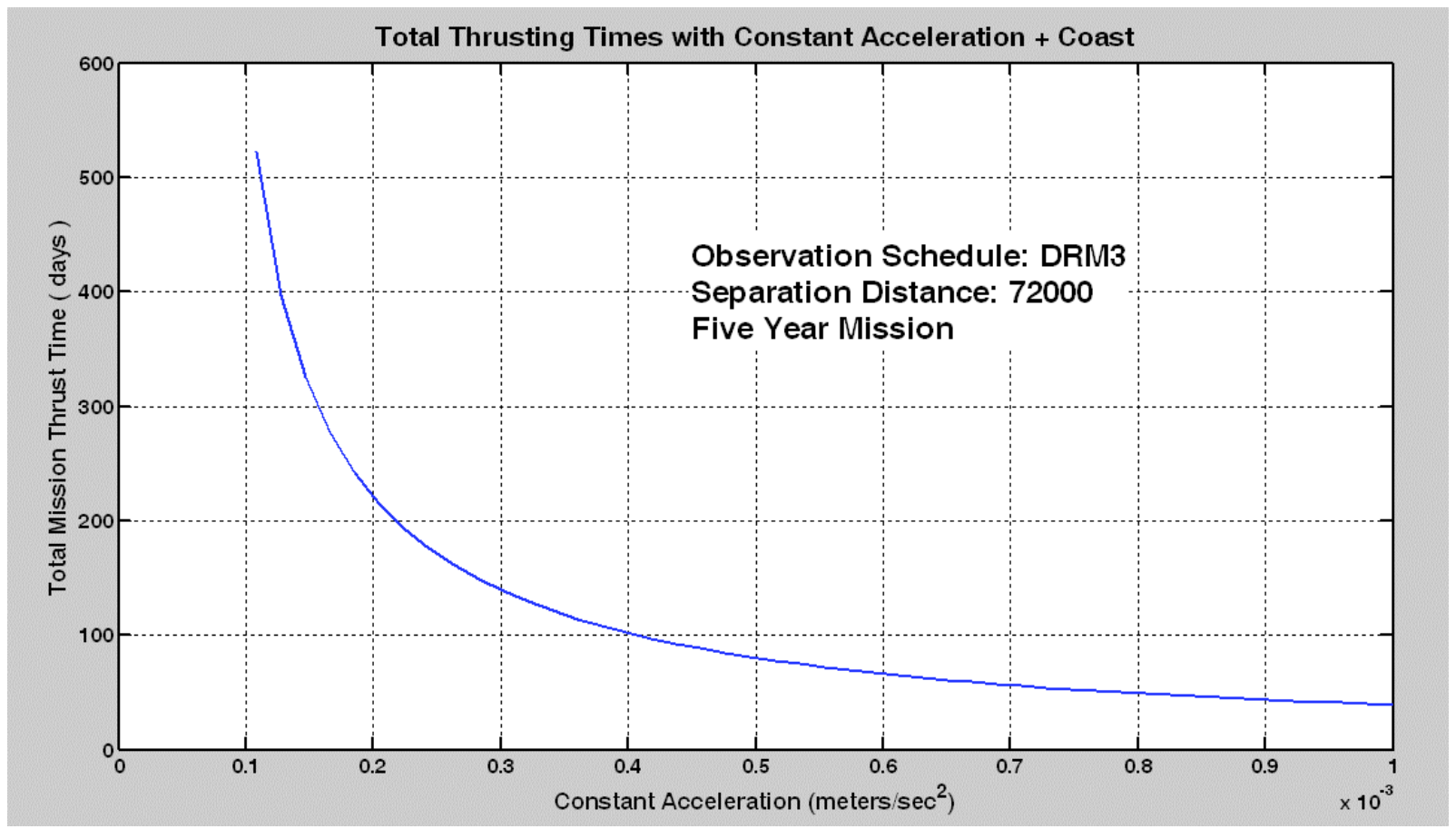

Figure 4. Thrusting time with constant acceleration.

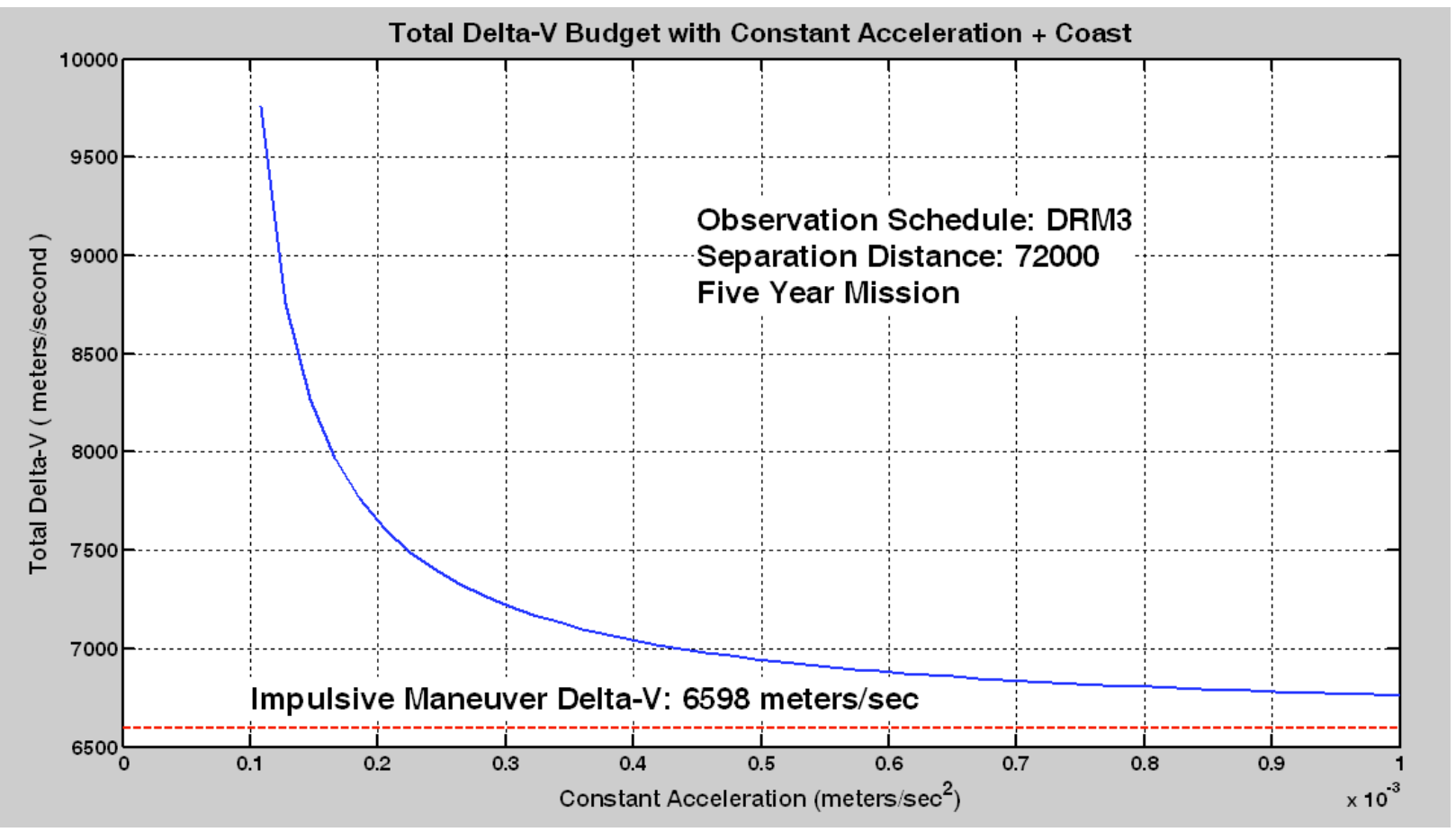

Figure 5. $\Delta V$ consumption for constant acceleration maneuvers. 
Table 4. Formation Flying Capability Requirements vs State-of-the-Art.

\begin{tabular}{|c|c|c|c|c|}
\hline & \multicolumn{3}{|c|}{ Figure of Merit } & \multirow[b]{2}{*}{ TPF-O related notes } \\
\hline Required Capability & Now (TRL 6-9) & Near-term demo & TPF-O & \\
\hline Measure relative position & $\begin{array}{l}2 \mathrm{~cm} \text { postproc } \\
\text { (over } 20,000 \mathrm{~km} \\
\text { measurement to } \\
\text { GPS transmitter) } \\
\end{array}$ & $\begin{array}{l}<50 \mathrm{~cm} \text { on-board, } \\
\text { real-time with } \\
\text { feedback to } \\
\text { formation control }\end{array}$ & $10 \mathrm{~km}$ & $\begin{array}{l}\text { Longer range required for TPF-O, but lower precision. } \\
\text { TRL } 4 \text { in TPF-O context, but can be transitioned to TRL } 6 \\
\text { using channel simulator without major cost or risk. }\end{array}$ \\
\hline $\begin{array}{l}\text { Measure } \mathrm{S} / \mathrm{C}-\mathrm{S} / \mathrm{C} \text { bearing } \\
\text { angles (combination of } \\
\text { relative attitude } \& 3 \text { axis } \\
\text { position) }\end{array}$ & N/A & 30 arcmin & 1 arcmin & $\begin{array}{l}\text { TRL } 3 \text { in TPF-O context, but depends on architecture. } \\
\text { Can be covered through line-of-sight measurement. }\end{array}$ \\
\hline $\begin{array}{l}\text { Control relative position } \\
\text { through comm. link }\end{array}$ & $\begin{array}{l}\text { Rendez/Docking, } \\
<1 \mathrm{~m} \text { short range, } \\
\text { one way }\end{array}$ & $\begin{array}{l}2.5 \mathrm{~m} \\
\text { (collaborative) }\end{array}$ & $100 \mathrm{~km}$ & $\begin{array}{l}\text { Longer range required for TPF-O, but lower precision. } \\
\text { TRL } 4 \text { in TPF-O context, but can be transitioned to TRL } 6 \\
\text { using channel simulator without major cost or risk. }\end{array}$ \\
\hline Control S/C-S/C bearing angle & N/A & $10 \operatorname{arcmin}$ & $10 \operatorname{arcmin}$ & $\begin{array}{l}\text { TRL } 3 \text { in TPF-O context, but depends on architecture. } \\
\text { Can be covered through line-of-sight control. Relevant } \\
\text { technologies and testbeds exist to transition to TRL } 6 \text {. }\end{array}$ \\
\hline $\begin{array}{l}\text { Formation line-of-sight } \\
\text { Control }\end{array}$ & N/A & $10 \operatorname{arcmin}$ & $1 \mathrm{mas}$ & $\begin{array}{l}\text { Tall pole. TRL } 3 \text {. Near-term demo would require gravity } \\
\text { environment beyond LEO. }\end{array}$ \\
\hline $\begin{array}{l}\text { Inter-S/C Communication } \\
\text { Rate }\end{array}$ & $\begin{array}{l}300 \mathrm{Mbps} \\
\text { TDRSS }\end{array}$ & $\begin{array}{l}10-1,000 \mathrm{Kbps} \\
<20 \mathrm{~W}, 20 \mathrm{~kg}\end{array}$ & $500 \mathrm{kbps}$ & $\begin{array}{l}\text { Longer range required for TPF-O, but lower precision. } \\
\text { TRL } 4 \text { in TPF-O context, but can be transitioned to TRL } 6 \\
\text { using channel simulator without major cost or risk. }\end{array}$ \\
\hline $\begin{array}{l}\text { Precision of time } \\
\text { synchronization }\end{array}$ & $\begin{array}{l}3 \text { ns GPS, on- } \\
\text { board real-time }\end{array}$ & $<1 \mu \mathrm{s}$ & $1 \mu \mathrm{s}$ & Range between spacecraft is only issue. \\
\hline
\end{tabular}

presents, in generic form, the key formation flying technology capability areas within TPF-O. The first column under the Figure of Merit caption identifies current capabilities at Technology Readiness Level (TRL) 6-9 in a general context, while the second column identifies an appropriate technological stepping stone for a nearterm space flight demonstration. The last Figure of Merit column represents the requirement for the TPF-O concept. The notes identify the relationship between the state-of-the-art technology status and the specific implementation required for TPF-O. Of note is the fact that most of the technologies required are at TRL 6 or better, but by definition in the TPF-O context, the TRL returns to 3, but for most cases, the transition back to 6 is straightforward and low in risk. The exception is the alignment control requirement, generically listed as "line-of-sight" control, where there is no existing technology implementation and the accuracy required is particularly stringent. Henceforth, the alignment sensing and control problem is identified as the tall-pole of the formation flying system development, and likely of the entire mission.

Finally, it is important to look across related missions which have flown - constellations, formation flying, and rendezvous and docking - and identify which have demonstrated key functional capabilities required for TPF-O. Table 5 maps the functional capability areas against the missions, where the black X marks represent successful demonstrations, blue represents demonstrations not yet performed, and red represents areas that were intended for demonstrate, but not successfully performed, either due to failure or early termination of mission. EO-1/LS7 (Earth-Observing 1/LandSat 7) was a low Earth orbiting demonstration within NASA's New Millennium Program (NMP) of formation flying of one vehicle relative to the other without direct communication and with measurements provided via bent-pipe through the ground. ST-5 (Space Technology 5) was a microspacecraft technology demonstration in NASA's NMP employing three spacecraft (without intersatellite communications or relative navigation/control). DART (Demonstration of Autonomous Rendezvous Technology) was a NASA mission intended to demonstrate key concepts in relative sensing and control for rendezvous and proximity operations, where several anomalies caused the mission to end up with a collision of the vehicles involved after successful performance of some of the mission objectives. ST7 DRS (Space-Technology 7, Disturbance Reduction 
Table 5. Functional Capabilities Demonstrated in Related Missions.

\begin{tabular}{|l|c|c|c|c|c|c|c|c|c|c|c|}
\hline Capability & EO1/LS7 & ST5 & DART & ST7 DRS & OE & ETS-7 & XSS-11 & GRACE & Cluster & SPHERES & TPF-O \\
\hline formation maneuvers & & & & & & & & & & & \\
\hline $\begin{array}{l}\text { Continuous formation } \\
\text { control }\end{array}$ & & & & & & & & & & & $\mathbf{X}$ \\
\hline Precise relative navigation
\end{tabular}

System) is a NASA New Millennium Program mission scheduled to fly on a European spacecraft, demonstrating highly precise measurement and control of a single spacecraft relative to an internal proof-mass. Orbital Express was a mission of the Defense Advanced Research Projects Agency (DARPA) to demonstrate key elements of rendezvous, docking, fuel transfer, and vehicle servicing. ETS-7 was a mission of the Japanese Space Agency (NASDA, now part of JAXA) that demonstrated autonomous rendezvous and docking of two spacecraft. XSS-11 was a mission of the Air Force Research Lab that demonstrated autonomous proximity operations, inspection, and circumnavigation of non-cooperative space objects. GRACE (Gravity and Climate Experiment) was a NASA gravity science mission that demonstrated precise relative range measurement between two spacecraft in low Earth orbit. Cluster is a European Space Agency mission that has demonstrated collective operation of a multispacecraft constellation. Finally SPHERES (Synchronized Position, Hold, Engage, and Reorient Experimental Satellites) are very small (few $\mathrm{kg}$ ) spacecraft that fly within the International Space Station, demonstrating a range of technology concepts relevant to formation flying and rendezvous and docking. These missions collectively have performed many of the relevant formation flying functional capabilities needed for TPF-O, however, little has been done to demonstrate any multi-vehicle capabilities in libration point and deep space orbits.

\section{SUMMARY}

A top-level formation flying system concept has been presented for enabling a free-flying telescope-occulter mission concept for finding distant planets. The functional breakdown of the formation flying system has been presented along with some of the key requirements and trades in the functional areas, such as relative navigation, intersatellite communication, and formation control. A concept for the most stringent mission requirement, that of measuring the telescope-occulter alignment to an inertial target, has been presented, and feasibility of the concept has been established. Results were presented for cumulative coasting time and cumulative thrusting time vs level of constant acceleration for a five year design reference mission using impulsive control for reconfiguration. The $\Delta V$ required for science mode maneuvers was determined to be just over $6.5 \mathrm{~km} / \mathrm{sec}$, which is high, but very representative of a fuel-enabled mission with frequent long-distance maneuvering. At this point, the analysis indicates feasibility of performing a telescope-occulter planet finding mission with engineering challenges, but minimal technology development. 


\section{ACKNOWLEDGMENTS}

Thanks go to Rick Lyon of NASA Goddard for the design of the alignment sensor system and to Rich Luquette, also at Goddard, for performing the formation control fuel analysis.

\section{REFERENCES}

1. Anne Long, David Kelbel, Taesul Lee, Dominic Leung, Russell Carpenter, and Cheryl Gramling. Relative navigation of formation-flying satellites. In Proceedings of the 1st International Symposium on Formation Flying Missions and Technologies, Toulouse, France, 2002.

2. David Kelbel, Taesul Lee, Anne Long, Russell Carpenter, and Cheryl Gramling. Evalution of relative navigation algorithms for formation-flying satellites. In 2001 Flight Mechanics Symposium. NASA CP-2001-209986, 2001.

3. R. J. Luquette and R. M. Sanner. A Non-Linear Approach to Spacecraft Formation Control in the Vicinity of a Collinear Libration Point. In AAS/AIAA Astrodynamics Specialists Conference, Quebec City, Quebec, Canada, 2003. AAS 03-113. 